\title{
Osos, conceptualizando sus masculinidades en Santiago de Chile
}

\section{Bears, Conceptualizing Their Masculinities in Santiago of Chile}

\author{
Daniel Benavides-Meriño \\ Universidad de Santiago de Chile, Santiago, Chile
}

\begin{abstract}
Resumen: La subcultura oso, amparada en el problema de una escasa producción teórica que la vuelve fenómeno casi desconocido en el país, hace al presente estudio pionero en la materia. La investigación tiene carácter exploratorio con una muestra no probabilística por conveniencia, conformada por seis participantes de entre 21 y 30 años, homosexuales y autoconcebidos "osos". Se realizaron entrevistas semiestructuradas, cuyos datos fueron analizados mediante transcripciones y posterior categorización. Los hallazgos señalan una masculinidad subordinada, teñida de heteronormatividad, afirmada en la heteroidentificación y en un cuerpo fetichizado en oposición a ciertos estereotipos de delgadez. La expresión de su condición es relegada a la vida nocturna y de entretención por experiencias teñidas de discriminación y vulneración de derechos. La sexualidad del oso en Santiago de Chile presenta una elevada actividad calificada como morbosa y promiscua. Relativo a las prácticas se mencionan la sexualización completa del cuerpo en desmedro de la genitalización. Paralelamente la figura del oso es concebida como significativamente intolerante y discriminadora respecto de otro tipo de masculinidades. La relevancia de los resultados radica en entregar un marco de análisis que permita comprender la realidad de nuevas identidades homosexuales masculinas ofrenciendo a las autoridades competentes la posibilidad de visualizar nuevos actores sociales en la frontera de la sexualidad masculina.
\end{abstract}

Palabras clave: oso, argot gay, heteronormatividad, fetichización.
Abstract: The bear subculture, based on the problem of a scarce theoretical production that makes the phenomenon almost unknown in the country, makes the present study a pioneer in the field. The research is exploratory with a nonprobabilistic sample for convenience, being made up of six participants aged 21 to 30 years old, gays and self-conceived bears. Semi-structured interviews were performed, whose data were analyzed using transcripts and subsequent categorization. The findings indicate a subordinated masculinity, tinted with hetero-identification, and affirmed in heteronormativity and in a fetishized body in clear opposition to some stereotypes of thinness. The expression on their condition is relegated to night life and entertainment experiences dyed with discrimination and violation of rights. Bear sexuality in Santiago of Chile is highly rated as a morbid and promiscuous activity. Practices concerning the complete body sexualization are mentioned in detriment of the genitalization. In parallel the figure of the bear is conceived as significantly intolerant and discriminatory with respect to other masculinities. Relevance of the results lies on providing a framework of analysis to understand the reality of new male homosexual identities offering to competent authorities the possibility of visualization of new social actors on the border of male sexuality.

Keywords: bear, gay argot, heteronormativity, fetishization.

Contacto: D. Benavides-Meriño. Obispo Umaña \#033, Depto. 1810 Torre Alerce, Estación Central. Santiago de Chile.Correo electrónico: daniel.benavides@usach.cl

Cómo citar: Benavides-Meriño, D. (2016). Osos, conceptualizando sus masculinidades en Santiago de Chile. Revista de Psicología, 25(2), 1-18.

http://dx.doi.org/10.5354/0719-0581.2017.44792 


\section{Introducción}

Históricamente se ha definido la masculinidad como repudio a la femineidad, sometimiento al cuidadoso y persistente escrutinio de la validación homosocial y como homofobia. La relación entre estas cuestiones hace emerger una serie de preconceptos que buscan naturalizar lo culturalmente creado por esquemas de referencia masculinos y femeninos en los que los hombres constantemente deben mostrar a sí y a otros que no son mujeres, bebés ni homosexuales (Kimmel, 1997).

$\mathrm{Al}$ respecto, David Gilmore (1994) señala que la masculinidad se construye desde un ideal individual y público como construcción cultural que se reproduce socialmente. Esta construcción es intervenida por distintas instituciones como la familia, el Estado, etc., las cuales moldean modos de habitar el cuerpo, de sentir, de pensar y de actuar (Faur, 2004). Así, el género está contextualizado en el tiempo y espacio histórico de las sociedades, lo que le exige una capacidad de adaptación para lograr la sobrevivencia del conjunto social (Diez, 2003). En otras palabras, el género cambia de una cultura a otra, en una misma cultura a través del tiempo, durante la vida de cualquier hombre individualmente y entre diferentes grupos de hombres según su clase, etnia y preferencia sexual; lo que demuestra el aspecto dinámico de la masculinidad (Kimmel, 1992).

Bermúdez (2013) señala que la hegemonía masculina comenzó a distar en susceptibles comparaciones del rol sexual masculino, puesto que no trata de roles, sino de un modelo de masculinidad "ideal" que no se corresponde con el de la mayoría de los hombres, necesariamente. En consonancia, Viveros Vigoya
(2002), con base en el estado del arte realizado por Kenneth Clatterbaugh (1997) respecto de la masculinidad, condensó los estudios en seis perspectivas para indagar y explicar la jerarquización de lo masculino. Una de ellas, de los grupos específicos, asegura que no existe universalidad en el concepto de masculinidad y que esta varía de acuerdo con las condiciones propias de cada sujeto, en las que convergen las homosexualidades, las etnias y las prácticas religiosas.

En este escenario cobra fuerza el concepto de masculinidades sistematizado por primera vez por Carrigan, Connell, y Lee en su artículo "Towards a new sociology of masculinity" (1985), en el cual intentaron oponerse a las teorías de roles e ilustrar la diversidad del universo varonil. En este se visibilizan masculinidades en tres categorías: hegemónicas, conservadoras y subordinadas. Esta última patenta la subordinación entre hombres, en la que los gays serían los principales discriminados al ser conceptualizados como femeninos (Bermúdez, 2013) o como masculinidades de segunda calidad (Faur, 2004).

En este punto del devenir teórico se reconoce como importante atender la otra vertiente que aporta interés al creciente estudio de la masculinidad: los gay studies. Estos estudios, con un claro intento de deconstrucción de los modelos masculinos en la frontera de la sexualidad, intentan reivindicar el derecho a las diferencias sexuales en los hombres, problematizando la tendencia asociativa de masculinidad-heterosexualidad y homosexualidad-femineidad (Viveros Vigoya, 2002). En ese sentido, los gay studies han mostrado que los comportamientos homo y heterosexual no están asociados a un sentido diferenciado de identidad sexual, necesariamente (Parker, 1998). 
La homosexualidad se ha concebido e identificado de variadas formas de acuerdo con el período histórico y el espacio sociocultural de referencia (Araya Andrade \& Echeverría Chavarría, 1998). Giddens (1991) entiende la homosexualidad como orientación de los sentidos o la actividad sexual hacia el mismo sexo; y Marmor (1980) como la atracción erótica preferencial definitiva a miembros del mismo sexo involucrándose en relaciones sexuales abiertas con ellos.

De acuerdo con Kenneth Plummer (1992), se presentan cuatro tipos de homosexualidad dentro de la cultura occidental actual. Una de ellas, homosexualidad como forma de vida, aplica a aquellos individuos que se autodeclaran homosexuales y que han convertido su homosexualidad en parte crucial de sus vidas, en tanto que pertenecen a subculturas gays, cuyas actividades homosexuales se integran a un estilo de vida concreto -mas se reconoce que la autodeclaración no excluye un comportamiento reservado si así el sujeto lo desea o se ve obligado de alguna forma a ello-.

Esta homosexualidad permite, dentro de una sociedad, la emergencia de comunidades o grupos distintos que tienen su propio territorio y modo de vivir (Araya Andrade \& Echeverría Chavarría, 1998). De acuerdo con Giddens (1991), esta diversidad cultural en el seno de una sociedad es lo que se conoce como subcultura, la que, según Kates (1998), se define como ideología coherentemente articulada de significados, creencias y comportamientos, además de ser una forma compleja de interacción y organización social. En el caso de los homosexuales, Sullivan (1996) menciona que la subcultura gay ha utilizado la subversión para resistir a los enérgicos sistemas que la oprimen. Como medios de subversión destacarían el libertinaje, la ironía y la risa, los cuales harían las veces de desprecio por las normas sexuales de género -como el cultivo al travestismo- y el uso distorsionado de la lengua (Trevisan, 2000).

Frente a esta práctica social de relaciones sexuales entre varones, se ha construido un estilo de vida primero y una subcultura después, que ha logrado legitimarse frente a los dispositivos del control social formal. Estas cuestiones constituyen sus mayores éxitos en lo político, mientras que en lo social se configura una identidad social específica: la identidad gay (Guasch, 2000).

Nunan y Jablonski (2002) señalan que, a diferencia de otras identidades, en el caso homosexual, el concepto permite la consideración de diferencia de deseos, aun cuando se compartan experiencias similares de marginalidad social y un sentimiento común de diferencia sexual (Green, 1999). Por tanto, mientras la subcultura homosexual permite que muchos homosexuales se deshagan de las etiquetas impuestas desde el exterior, esta posibilita también la subdivisión de la misma en grupos más pequeños que tienden al autoetiquetado.

Una de las subculturas homosexuales que mantiene particular relación con la representación de la masculinidad es la bear - de los osos- que para Wright (1997) aparece como síntoma del resurgimiento de la vida gay estadounidense en la década de 1980. Luego de la conmoción causada por la propagación del sida en esos años -en que el varón gay fue el más afectado- los osos surgen con una propuesta ética a la recuperación de la autoestima para continuar con la tradición de identificación masculina gay y la defensa de los valores de equidad. 
Esta subcultura, a modo de estrategia, supone una forma de cuestionamiento a una presunta naturaleza masculina a partir de una reivindicación artificial basada en el exceso. Sáez (2005) plantea que esta subcultura produce un efecto paradójico en las representaciones simbólicas y políticas de lo masculino, puesto que intenta construir un cuerpo e ideas "naturales" con base en unos valores tradicionales de la masculinidad como la barba, el vello corporal, el cuerpo fuerte, grande u obeso, y la virilidad.

Pero, ¿qué es un oso?, ¿cómo luce un oso? Hennen (2005) reconoce que muchas de sus respuestas hacen referencia al cuerpo como intento de describir la fisionomía típica de un oso. En esto destaca George Mazzei con su artículo pionero para la revista The Advocate llamado "Who's who in the zoo?" que caracterizaba a los homosexuales como siete tipos de animales, incluyendo los osos. En este se describe al oso como barbón, risueño, hogareño, amoroso y fiel, además de ser "... generalmente tipos corpulentos, robustos, con reminiscencias de un leñador o ex jugador de rugby” (Mazzei, 1979).

La forma de taxonomía física más aceptada a nivel mundial se relaciona directamente con la edad, el rol sexual, la actitud, la vellosidad y la masa corporal. En este ámbito destaca el Natural Bears Classification System (Sistema de clasificación natural de osos) inventado por Donahue y Stoner (como se citó en Gutiérrez, 2004). Además, en Recursos para Osos. Los Códigos de los Osos (Donahue \& Stoner, 2001) existen subtipos que patentan graduación de presencia para cada uno de ellos -que no representan una valoración de los mismos-. En consonancia se evoca al cuerpo del oso como el principal parámetro de masculinidad, lo que lleva el porte a la actitud demostrada por los sujetos en la presentación de sí mismos elaborada frente a otros (Blázquez \& Liarte, 2013). Esta particular forma de vivir y entender la masculinidad no se concibe como forzada ni impostada, sino como natural, lo que llamaría la atención del varón homosexual oso a modo de cuasiontología del ser masculino (Blázquez \& Liarte, 2013). Esta observación es concebida por Sáez (2005) como una representación que intenta replicar lo natural, en la que el oso juega con una presunta naturaleza salvaje que constituye luego una masculinidad idealizada y animal.

Se está, por tanto, frente a una estilización de la conducta, proceso del que también participa la masculinidad heterosexual, y cuyos códigos se aprenden desde el nacimiento y que continuamente se repiten, marcando la experiencia subjetiva de la masculinidad. Es más, Sáez (2005) sentencia que "la heterosexualidad es uno de esos rasgos que constituyen la masculinidad ideal. Lo interesante de los... osos es que utilizan los códigos masculinos pero al final se produce una traición, puesto que no son hombres 'de verdad' porque son gays" (p. 145).

Otra posible lectura respecto de esta naturalidad plantea la integración del modelo heterosexual dominante, lo que implica la recuperación subsecuentemente de su factor plumófobo ${ }^{1}$-además de misógino y lesbófobo-, que consiste en acusar a "las locas" de dar una imagen ridícula de los gays. De este modo, se reivindica una masculinidad "normal" que busca la aceptación del colectivo heterosexual (Sáez, 2005). En suma, la masculinidad oso no se concibe como reacción contra la masculinidad en sí, puesto que pareciera estar

\footnotetext{
${ }^{1}$ Se desconoce existencia de una definición conceptual oficial, mas se entiende como miedo y/o rechazo a los gays afeminados.

${ }^{2}$ Término peyorativo para referirse a un gay femenino.
} 
más interesada en la revisión de la masculinidad convencional, sin resignificarla.

Lo anterior denota que la subcultura oso ha sido fuertemente influenciada por la masculinidad hegemónica $\mathrm{y}$, en menor medida, por la heteronormatividad, pero igualmente se reconoce la resistencia e innovación sexual dentro de esta, lo que señala una recuperación parcial del cuerpo y un descentramiento -también parcial- del sexo fálico. En este orden de cosas se produce un interesante cruce referencial de la propuesta oso con la leather-subcultura referente a la conducta fetichista de mirar, oler y sentir ropa y objetos sexuales de cuero-, ya que se reconoce que ambas se volvieron aliadas y permitirían el libre cruce de fronteras en la flexible ubicación identitaria (Gutiérrez, 2004).

En "Masculinidad, piel y rudeza: subcultura leather" (HAZLO.mx, 2014) se expresa que los elementos que se identifican con la virilidad en ambas propuestas dicen relación con uniformes, profesiones y ambientes exclusivos de varones -como el ejército o la marina-, por lo que se copian y se adaptan estos referentes como cuestionamiento a los mismos. Sin embargo, se alejan del estándar heterosexual predominante al practicar juegos sexuales descentrados del pene erecto y la eyaculación (Sáez, 2005). Este mismo autor señala: "Esta exhibición... de la vulnerabilidad genital supone un giro histórico en la representación de la masculinidad, donde el presunto falocentrismo de las prácticas gays se ve cuestionado radicalmente" (p. 144). Paralelamente en los osos conviven prácticas institucionalizadas como el abrazo del oso y el nuzzling ${ }^{3}$ de piel, que dan cuenta de

\footnotetext{
${ }^{3}$ Práctica de tocar, frotar o presionar algo o alguien con suavidad, generalmente con movimientos repetitivos.
}

que la sensualidad en los osos desafía los supuestos hegemónicos de la sexualidad masculina (Gilligan, 1982), ya que se reivindican como formas de socialización afectivas entre hombres en lugar de meramente sexuales. La camaradería en lugar de la competencia patenta un tipo de masculinidad propio de esta subcultura que se ausenta en otras de orden homosexual (Hennen, 2005).

Hennen (2005) enfatiza la forma en que la sensualidad divide la práctica sexual del oso entre sexual y sensual, donde esta segunda arista ayudaría a fomentar una actitud más responsable respecto al sexo. Esto es significativo, porque demuestra que para la mayoría de los osos el fomento de la cultura sexual con penetración es una elección consciente y deliberada. Por tanto, "la sexualidad entre los osos es sensualidad primero" (p. 38).

En consecuencia, emerge el debate en torno a las consecuencias que esta subcultura ha tenido en la resolución y consolidación de falsos binomios que la presente investigación articula entre género, sexualidad e identidad para reconocer la importancia del cuestionamiento de la performatividad de género en lo referente al surgimiento de nuevas masculinidades. Paralelamente recalca la relevancia de análisis y/o transformación de modelos de masculinidad donde la categoría género sigue siendo central en Chile para segmentar y jerarquizar a los grupos sociales, puesto que cumpliría una función legitimadora que permite sostener desigualdades.

En lo nacional, probablemente, los discursos públicos hayan cambiado, pero en un nivel latente se mantienen actitudes negativas hacia los homosexuales, las que surgen en contextos menos regulados o que implican respuestas no deliberadas. Este efecto implica el desvío de los roles 
de género y no la homosexualidad en sí, la que sería sancionada (Barrientos \& Cárdenas, 2013).

En este sentido, ¿cómo los sujetos autoconcebidos homosexuales osos conceptualizan sus masculinidades ante la contradicción de ser varones gay sin perder los privilegios de la masculinidad hegemónica, e igualmente en contraposición a la identidad gay dominante? Para ello se considera necesario el cumplimiento de los siguientes objetivos específicos: identificar las significaciones que hacen de su rol de género por medio de los principales símbolos y clasificaciones propios de la subcultura; indagar en torno a la vinculación emocional y sexual respecto a otros gays y osos; y conocer la socialización de sus masculinidades osos entre sí y con otras masculinidades gays.

Esto resulta relevante para la psicología desde el prisma de los estudios de la homosexualidad, puesto que entrega un marco de análisis que permite comprender la realidad de nuevas identidades homosexuales masculinas como fenómeno emergente en la realidad local. Lo anterior constituye una necesidad, ya que ofrece la posibilidad de proveer insumos a las autoridades competentes para enfatizar debates en la agenda pública referente a la temática de las minorías sexuales, de manera de que se la oriente no solo a aspectos como la desestigmatización e integración social, sino también a la visibilización de nuevos actores sociales en la frontera de la sexualidad masculina.

Este estudio constituye un primer acercamiento a la conceptualización de este tipo de masculinidad homosexual en Chile, cuyo estado del arte latinoamericano es aún incipiente. Además, compone una aproximación al estudio de los procesos de socialización de género en hombres que expresan una masculinidad no hegemónica en contextos donde se la valora; vale decir, en un contexto social con una legislación que resguarda y protege sus derechos, pero en que viven todavía su sexualidad en secreto debido a la discriminación.

\section{Método}

\section{Diseño}

De carácter exploratorio (Hernández, Fernández, \& Baptista, 1998), esta investigación representa uno de los primeros acercamientos al fenómeno de la subcultura oso en cuanto forma de representación, identificación y pertenencia de varones homosexuales en Santiago de Chile; esta siguió una lógica descriptiva, al especificar las propiedades importantes del fenómeno en cuestión donde la subjetividad se torna central de acuerdo con lo señalado por Dankhe (1989). A través de esta se intentó generar conocimientos que permitan incrementar las investigaciones en el tema y construir una mirada desde los mismos participantes por medio de su propia narrativa y discurso, centrado en su conceptualización de las masculinidades homosexuales oso con base en los relatos vinculados al cuerpo, práctica sexual y socialización con otras masculinidades gays.

\section{Técnica de producción de información}

Se realizó mediante entrevistas semiestructuradas que, gracias a su flexibilidad y dinamismo, permiten adecuarse a los cambios producidos en la interacción entrevistador-entrevistado y atender particularidades propias del discurso de este último dirigidas a la comprensión de sus perspectivas respecto de sus vidas, experiencias o situaciones, tal como las expresan (Taylor \& Bogdan, 1986). 


\section{Participantes}

Se consignan seis participantes seleccionados de manera no probabilística por conveniencia al realizarse dirigida $\mathrm{o}$ intencionalmente (Martín-Crespo Blanco \& Salamanca Castro, 2007), cuyas edades oscilaron entre 21 y 30 años, autoconcebidos homosexuales osos y con características teóricas definitorias de lo considerado propiamente ursino: masculinidad demostrada en comportamiento y fisionomía típica.

El estudio siguió una estrategia de informantes clave y bola de nieve (Taylor \& Bodgan, 1986) que resulta útil cuando el objetivo persigue comprender la red social de un cierto grupo o comunidad (Flick, 2004). Se está en consonancia con las características de la población a la que apunta la investigación, a la vez que se considera igualmente lo exploratorio de la temática atendida.

Tabla 1

\begin{tabular}{cc}
\multicolumn{2}{c}{ Profesión/ocupación de los participantes } \\
\hline Edad & Profesión/Ocupación \\
\hline 21 & Fotógrafo \\
29 & Director de arte \\
26 & Publicista \\
27 & Ayudante de cocina \\
23 & Licenciado en Letras \\
30 & Magíster en Química \\
\hline
\end{tabular}

\section{Estrategia de análisis}

Se utilizó la categorización como estrategia de análisis, ya que permite organizar y presentar la información de acuerdo con algún patrón o regularidad emergente (Iñiguez \& Antaki, 1998) de modo que se destaque la importancia que se deposita no solo en las palabras del enunciado, sino también en los supuestos culturales a la base del mismo (Kulka, 2000). El en- foque se centra tanto en el discurso de los participantes como en la influencia e incidencia de la realidad homosexual nacional que esto conlleva.

Estas categorías fueron construidas considerando las tres dimensiones de la unidad de análisis, además de otras que emergieron del contenido relevante para la investigación presente en las variadas entrevistas que hizo las veces de catalizador en la adquisición de la identidad oso. A saber: 1) conceptos de masculinidad, homosexualidad y masculinidad oso; 2) sexualidad y erotismo; 3 ) socialización intra y extragrupal; 4) salida de clóset; 5) provocación en el vivenciar oso; y 6) rol sexual.

Una vez categorizadas todas las entrevistas de acuerdo con las subcategorías, se procedió a capturar todos los códigos que respondían a cada concepto, los cuales se pudieron contrastar con la teoría y producir luego los resultados.

\section{Consideraciones éticas}

Para resguardar los aspectos éticos de la investigación y en conformidad a la legislación vigente se consideran las siguientes normativas: a) Ley 19.628 (2012), sobre protección de la vida privada; b) Ley 20.120 (2013), sobre la investigación científica en el ser humano, su genoma y prohíbe la clonación humana; y c) Normativa institucional de la Comisión de ética de la Vicerrectoría de Investigación y Postgrado de la Universidad de Santiago de Chile. En conformidad a ello se presentó un consentimiento informado a los participantes para constatar formalmente su deseo de participar por medio de la firma del mismo.

El estudio de campo de esta investigación se realizó con la conformidad del Comité 
de Ética de la Vicerrectoría de Investigación, Desarrollo e Innovación de la Universidad de Santiago de Chile.

El compromiso ético y político fue declarado desde el primer contacto con los participantes. Vale decir, que la presente investigación tiene un claro compromiso social que pretende no solo averiguar y recopilar información, sino promover la discusión de aspectos sociales que se mantienen naturalizados y que resultan, acorde a los tiempos actuales, susceptibles de un necesario cuestionamiento $-\mathrm{y}$ deseada modificación- para lograr un funcionamiento dotado de mayor libertad en las personas.

\section{Resultados}

\section{Conceptos de masculinidad, homose- xualidad y masculinidad oso}

Se apunta que, conceptualmente, la masculinidad destaca por ser un término ambiguo, altamente subjetivo de conceptualizar, pero claramente tendiente a la oposición de femineidad. Aun así, se identifican los aspectos físicos y actitudinales que hacen masculino a un hombre. Respecto a los primeros, predomina un gran volumen corporal -en estatura, grasa y/o músculo-, vello facial/corporal y una voz grave; sobre los segundos, la protección a otros, estoicismo y ausencia de conductas como autocuidado, apreciaciones estéticas y "mujereo"4.

La homosexualidad es significada como atracción/preferencia/condición hacia personas del mismo sexo donde el encuentro sexual es decidor para definirse o no como homosexual. Su expresión en

\footnotetext{
${ }^{4}$ Dinámica referida al trato en femenino para y entre hombres -generalmente entre homosexuales- con finalidad de burla, entretención o menosprecio.
}

Santiago de Chile transita desde el temor/recelo -marcado por la discriminación y la vulnerabilidad de derechoshasta una libre expresión naturalizada en espacios ligados preferentemente a la vida nocturna.

De esta manera, los participantes convergen en la idea de diferencias inexistentes entre masculinidad homosexual y heterosexual, pues ambas serían construidas por el devenir cultural en el que sus mismos adscritos velarían por mantenerla. Es menester mencionar que la subyugación de lo femenino sería característica de tal cuestión.

La identidad oso fue señalada o sugerida por terceros la mayoría de las veces - parejas, amigos y/o colegas- a modo de heteroidentificación. Sin embargo, el primer acercamiento a la cuestión fue propiciado por cuestionamientos personales y/o despertar sexual ligado a la figura erótica del cuerpo hipermasculino en la adolescencia.

Los participantes configuran su identidad oso como un arma de doble filo que propicia garantías sociales en el capital sexual en modalidad de atractivo y cantidad de encuentros y, al mismo tiempo, como instancia que coarta y limita el libre tránsito entre conductas masculinas y/o femeninas desde el prisma de la heteronormatividad. Destaca la identidad oso como camuflaje social que permite la entrada y salida del clóset a voluntad, mas siempre dependiente del contexto donde el sujeto se inserte para cada posibilidad.

\section{Salida del clóset}

Este modismo estadounidense -entendido como la declaración voluntaria y pública de la homosexualidad-resulta relevante al 
ser considerada por los entrevistados como un episodio inicial y crítico en la construcción de sus identidades homosexuales. De esta forma, se consigna como quiebre que propició la búsqueda de una identificación que acunara la mezcla de visibilización de su homosexualidad con la performance genérica masculina en desmedro de lo propiamente femenino adscrito a la condición estereotipada del twink ${ }^{5}$.

\section{Provocación en el vivenciar oso}

Los entrevistados enfatizan en la exposición que el oso da al cuerpo, por cuanto adopta una actitud desafiante y transgresora respecto de una imagen corporal hipermasculina; esto contrasta con una actitud femenina y/o queer con objeto de visibilizar un tipo diferente de masculinidad.

\section{Sexualidad y erotismo}

En las entrevistas se evidencia, también, una sexualidad elevadamente activa definida como hipersexual y morbosa, en la que el sexo casual y la promiscuidad son la tónica experiencial. Pese a reconocerse preferencias por un cuerpo oso al pensar en una posible pareja sexual, ello no sería determinante al momento de concretar encuentro alguno, pues manifiestan tener una diversa gama de parejas previas.

Entre las características personales que los entrevistados creen que resultan atractivas para otros homosexuales destacan como factores físicos el vello corporal/facial, la masa muscular y accesorios como tatuajes y piercings -calificados como fetiches-. Aspectos como la rudeza, autoconfianza y desinhibición serían

\footnotetext{
5 Varones homosexuales de aspecto joven, delgado, ectomorfo; usualmente lampiños y rubios. También puede referir al acrónimo: Teenage, White, Into No Kink,
}

las predilectas como actitudinales, principalmente en la performance sexual. Pese a esto, atribuyen su apariencia oso solo como deseable para otros osos, en tanto que los no osos serían reticentes/renuentes al contacto sexual con ellos.

Relativo a las prácticas se mencionan la sexualización completa del cuerpo dotando de significativo erotismo los encuentros por medio de caricias, abrazos y besos. Del mismo modo, se cree que existe alta convergencia con lo leather y los juegos de rol, pese a reconocer escasa y/o nula realización de prácticas de dicha índole en el plano personal.

\section{Rol sexual}

Es entendido como la preferencia por otorgar y/o recibir la penetración durante el encuentro sexual y la adopción de una posición dominante o receptora-activo y pasivo respectivamente- . Se observa una fuerte tendencia a adscribir una performance genérica que dota de cualidades femeninas a los pasivos lo que tiene como consecuencia que se les valore aún más peyorativamente cuando estos se identifican como osos.

\section{Socialización intra y extragrupal}

La socialización dentro de la subcultura oso estaría fuertemente influenciada por una constante comparación a nivel individual y (auto)discriminación a nivel grupal, observada en la tendencia de medir los propios atributos típicamente osos con los de un tercero a modo de contrastar cualidades desde un prisma valorativo. En contraposición, se evidencia una fuerte preferencia entre osos de relacionarse con similares para propiciar un sentimiento de pertenencia y aumentar el propio atractivo sexual en contextos sociales. 
Las dinámicas serían realizadas preferentemente en el contexto de fiesta en reconocidas discotecas de la capital, las cuales organizarían eventos temáticos para este grupo en los que la trascendencia de las relaciones emergentes sería dependiente de la naturaleza misma de cada vinculación. No se evidencia existencia de actividades consideradas propias de oso, pero destacan los saunas y fiestas privadas con objeto de concretar encuentros sexuales, y actividades $\mathrm{o}$ eventos a beneficio realizadas esporádicamente por agrupaciones de osos (Bear44 y Boys \& Bears) ${ }^{6}$.

Respecto de los códigos de relación se menciona conocer y manejar las diferentes clasificaciones de la subcultura, mas no utilizarlas de forma regular e igualmente experimentar recelo por quienes las usan en desmedida. Si bien las califican de innecesarias y segmentarias, serían útiles para una comunicación expedita vía electrónica en modalidad de presentación personal por medio de aplicaciones para ligar.

Resultan antagónicas las percepciones de existencia o no de una subcultura oso en Santiago de Chile, pues, por una parte, se evidencia un grupo concreto de homosexuales circunscritos a las actividades recreativas donde el reconocimiento entre ellos sería evidente. Por otra, la masificación del movimiento se concibe incipiente y profuso de adscritos que no hacen de su homosexualidad un estilo de vida, debido a que la segregación y autodiscriminación limitarían tal cuestión.

\footnotetext{
${ }^{6}$ Agrupaciones de y para osos, que nacen a inicios de 2010. Su objetivo es aportar en la promoción y difusión de la cultura oso y ser un punto de encuentro entre quienes sientan atracción al movimiento. Para esto congrega a los usuarios para generar espacios de difusión organizando principalmente eventos y fiestas.
}

Finalmente, las dinámicas extragrupales son conceptualizadas como más sinceras y honestas, ya que la performance masculina puede flexibilizarse sin temor al prejuicio ni al rechazo. Se explicita que las amistades no osos conceptualizan a los osos como hombres físicamente eróticos y de personalidad cálida. Cabe mencionar que la primera observación es realizada por hombres homosexuales sean estos osos o no, mientras que la segunda es otorgada preferentemente por hombres y mujeres heterosexuales.

\section{Discusión}

Los sujetos autoconcebidos osos en Santiago de Chile consignan una masculinidad reafirmada en la apariencia y en la que la actitud es relegada a un plano secundario. En este sentido resulta contradictorio a la teoría el que se identifiquen como osos y no se visualicen necesariamente como masculinos al remarcar en sí rasgos corporales viriles que rápidamente se siguen de ensalzamientos de comportamientos en ellos considerados tradicionalmente como femeninos.

$\mathrm{Si}$ bien se reconocen factores comunes en los entrevistados -condición sexual, grupo etario, raza, nivel socioeconómico-, se enfatiza el aspecto dinámico de su masculinidad oso que depende del devenir particular de cada sujeto, en el que destacan las labores académicas y microactivismos referidos al género y sexualidad como posibles causas de las diferencias entre ellos. Esto converge con los planteamientos de Kimmel (1992), quien refiere variaciones en las definiciones de masculinidad a nivel cultural e individual.

Lo anterior permite reflexionar sobre lo que Connel (1987) denominó masculinidades subordinadas en las que destaca al 
varón gay como principal discriminado al ser considerado como femenino o portador de una masculinidad de segunda calidad. Ello se manifiesta en la vivencia teñida de temor y recelo de la sexualidad y el afecto en público -extrapolable a lo homosexual en general-, siendo esta relegada a espacios construidos fuera de la normatividad social como la vida nocturna y aplicaciones de ligue.

El cuerpo ocupa un lugar privilegiado en la construcción de la sexualidad al estar repleto de significaciones y reflejar un sistema de símbolos y, por tanto, resulta fundamental en la construcción identitaria y en el vivenciar sexual (Viveros Viveros \& Garay Ariza, 1998). En este sentido los resultados convergen con los planteamientos de Blázquez \& Liarte (2013), quienes erigen el cuerpo como el componente clave en la conformación identitaria del oso, al expresar que una serie de elementos corporales son requeridos para definirse como tal.

Es importante señalar la presencia de una asociación constante entre el volumen corporal con el cariño y la ternura, lo que deriva en que a los participantes se les visualice como individuos altamente confiables y cariñosos. Estos valores son defendidos por la propuesta oso, pero también extrapolables a la cotidianeidad heterosexual de la figura del marido, "el gordo", lo que hace pensar el cuerpo oso como estereotipo corporal que escapa a una condición sexual particular.

La sexualidad del oso en Santiago de Chile se presenta como una práctica de elevada actividad y que se caracteriza como morbosa y promiscua con una performance altamente erótica. Asimismo se destaca por la capacidad para entablar lazos afectivos con una disposición sensual también conocida como bonding homoerótico
(Hardman, 1993). Esta puede manifestarse en modalidad de atracción inconsciente, en expresiones afectivas, en fantasías y/o consumarse en el encuentro físico y disfrute de los cuerpos.

Con respecto a las prácticas se mencionan la sexualización completa del cuerpo en desmedro de la genitalización, lo que dota a los encuentros de un erotismo significativo. Esta realidad coexiste en armonía con las investigaciones de Gilligan (1982) y Hennen (2005), quienes plantean que la sensualidad y dispersión completa del placer por el cuerpo se presenta en desmedro del énfasis fálico. Pese a esto, la innovación y evolución sexual teórica, que la propuesta referencial leather y que el mismo autor señalara, se remiten al imaginario de los participantes al percibir la cuestión del cuero y sadomasoquismo como propia de osos, pero ajena a su vivenciar y al de terceros conocidos.

Por su parte, la adopción de roles sexuales en las relaciones ursinas resulta contraria a los resultados de Blázquez \& Liarte (2013) en la identificación de los mitos respecto de la edad y concepción de la gordura. Los participantes refieren una noción fuertemente influenciada por la heteronorma al referir automáticamente al rol pasivo una performance genérica femenina y una valoración peyorativa de la misma. Se señala una escasa proporción de osos pasivos al hallarse incompatible la hipermasculinidad oso con tal preferencia.

Igualmente, un hallazgo importante es la significación otorgada por los participantes a la homofobia internalizada, proceso que se sustenta en la construcción e internalización de roles de género rígidos (Delgado et al., 2016). Debido a esto, este tipo de homofobia es una de las cau- 
sas de la discriminación y autodiscriminación que existe entre osos. Los osos han aprendido a valorar la virilidad a través de un desprecio y un rechazo hacia los hombres afeminados. Por esta razón, los participantes son conscientes de la discriminación a los gays femeninos, ya que estas figuras subvierten las expectativas de género socialmente esperadas lo que intensifica la discriminación y la permanencia de la socialización de género dominante (Movimiento Unificado de Minorías Sexuales, 2006).

Referente a la socialización de la masculinidad oso entre sí y con otras masculinidades homosexuales, los resultados exponen contradicciones a nivel teórico y práctico. A nivel intergrupal, se enfatiza una percepción de comparación tendiente a evaluar desde un marco valorativo los propios atributos viriles con los de un tercero; emulando la competitividad en la lucha por el capital sexual tasado en el atractivo físico. Ello resulta antagónico a los postulados de Sáez (2005), quien señala que la vinculación entre homosexuales osos se erige sobre una política de defensa valórica basada en la equidad.

Paralelamente la figura del oso es concebida como significativamente intolerante y discriminadora respecto de otro tipo de masculinidades y, por tanto, de homosexuales ajenos al movimiento. Se enfatiza una socialización altamente segmentaria y (auto)discriminadora, la que es ejemplificada con las actividades y lugares frecuentados donde la estratificación es evidente en el espacio de la discoteca. Incluso su propia simbología es percibida como marginadora al observarse un desapego de la tradicional homosexualidad por considerarla en exceso femenina.

Sobre esta última cuestión, los resultados dan cuenta de un considerable manejo de las clasificaciones en la totalidad de los participantes, mas, contrario a lo que evidencia la teoría, estas son percibidas negativamente al estar teñidas de un fuerte juicio valórico y de una función alienante. Ello se sustenta sobre la percepción de categorías que instauran una determinada forma de ser, verse y actuar que puede o no ser concordante con el carácter.

El oso que expresa sus emociones colectivamente y es cariñoso con sus símiles a través del contacto amistoso y la camaradería (Gutiérrez, 2004) se contrapone a la percepción santiaguina de la figura de un oso discriminador y poco tolerante hacia las masculinidades. Resulta susceptible elucubrar un excesivo apego al modelo heterosexual dominante -con su correspondiente factor plumófobo y misóginocomo causa desencadenante de un implícito deseo de aceptación del colectivo heterosexual (Sáez, 2005) que dificulta la emergencia de un gay que cuestione la hegemonía y a la vez perturbe los códigos binarios de género. Asimismo, y según lo planteado por Wright (1997), los osos se comprometen con la masculinidad hegemónica, mas no buscan una respuesta alternativa a la misma.

\section{Conclusiones}

La identidad homosexual oso, como parte de una identidad masculina, está ligada a procesos interactivos y de heteroidentificación al interior de su propia colectividad donde el cuerpo propicia la emergencia de la categoría oso y la actitud la mantiene (Giménez, 1996). La masculinidad oso, además, no solo tiene un carácter relacional, sino que también emerge como consecuencia contestataria de una identidad homosexual que fue imponiendo un estilo de vida constringente en donde el cuerpo delgado, joven 
y lampiño representa uno de los más apreciados rasgos culturales.

Previo al trabajo de entrevistas se planteó que la masculinidad homosexual oso: 1) emerge del doble cuestionamiento a la masculinidad hegemónica con una performance que muestra la fragilidad de la misma y a la relación asociativa entre homosexualidad y afeminamiento; 2) se apropia del cuerpo como espacio de diferenciación y oposición ante un estilo de vida gay dominante; y 3) enfatiza una socialización afectiva y amistosa entre gays por un esfuerzo humanizador de la misma en desmedro de lo meramente sexual. Además de corroborar los primeros y desestimar el último punto, la investigación ahondó en: 1) el modo de vivir que se pretende enfrentar; 2) las percepciones valóricas sobre las taxonomías corporales; y 3) las opiniones sobre la existencia o no de una subcultura oso en Santiago de Chile.

Respecto al primer objetivo, se señala que esta configuración de masculinidad no solo reproduce una división binaria de prácticas y discursos con respecto al género, sino que también busca distancia de todo indicio de feminización de la homosexualidad. De este modo, y de acuerdo a la categoría de género propuesta por Lamas (1996), los osos de la capital producen y reproducen relaciones tradicionales de subordinación y dominación entre hombres y mujeres, las cuales, si bien están físicamente ausentes, no lo están simbólicamente. Es así como los osos construyen diferencias que distinguen los sexos a través del uso de significantes de poder como símbolos culturales que evocan representaciones múltiples -"el cola fuerte", "el maricón pluma"-, conceptos normativos -"ser masculino"- o de identidad -"ser gay oso"-.
A este respecto se observa una masculinidad homosexual fuertemente influenciada por la hegemónica, en la que un prisma heteronormativo merma la revisión de la masculinidad convencional por lo que se resignifica escasamente. Así, los varones oso en Santiago de Chile se definen desde una perspectiva esencialista de la masculinidad (Weeks, 1998), sin considerar las diferencias de género como producto de construcciones sociohistóricas, o bien, a través de una incorporación acrítica de la propuesta ursina en la realidad local. Por lo mismo, sus atributos se sostienen y refuerzan por mandatos sociales que son internalizados y forman parte de su identidad, en otras palabras, expresan esa masculinidad dominante que es su referente.

Sobre el segundo objetivo, referido al cuerpo y práctica sexual, se piensa que más que un abogar por el cuerpo grueso, gordo y velludo, se adopta una actitud de acercamiento y exacerbación a lo tradicionalmente considerado masculino. Esto a modo de reorientación del deseo hacia la imagen masculina que se ha mercantilizado en forma de fetichización del cuerpo, por medio de una cadena de ofertas y demandas. Pese a que el cuerpo oso escapa de los parámetros de belleza actuales, se hipotetiza la existencia de un mercado sexual corpo-subcultural entendido no solo como un cuerpo individual, sino que inserto en una estructura de taxonomías corporales que le aseguran una comodidad ficticia y le proporcionan alimento al ego.

Le Breton (1995) afirma que el cuerpo, en la modernidad individualista y narcisista, funciona como un límite fronterizo, pues se convierte en refugio y valor último del individuo y, en consecuencia, se transforma en una propiedad y necesidad 
de primer orden, objeto de todas las atenciones. A este respecto, al cuerpo oso se le exige que pueda mantener y acrecentar el capital sexual bajo la forma de seducción. Se habla de una selección, jerarquización y codificación del cuerpo y la conducta como principales rasgos subculturales en la delimitación de fronteras simbólicas en su interacción.

Subsecuentemente, la práctica sexual del oso se enmarca en percepciones jocosas de varones altamente eróticos y sexuales, en apariencia exentos de obligación a tener una vida sexual frecuente y constante disponibilidad. No obstante, y de acuerdo con su estrecha relación con una performance masculina significativamente heteronormada, es posible inquirir una incitación a la desatención de sus necesidades reales, tanto emocionales como físicas, entre estas, de su deseo sexual, por ser varones compelidos a poseer una sexualidad avasalladora como si más fuera sinónimo de mejor.

Cuestionar los convencionalismos de género únicamente en la intimidad de la construcción identitaria oso resulta una estrategia más necesaria que justa para contrarrestar los costos de salir de la norma. Esto, a su vez, les permite a los osos ser aceptados -en apariencia- por la sociedad y no ser configurados como completamente disidentes. Sin embargo, al interior de la subcultura se enarbola una actitud erótico-relacional con el objetivo tendiente a formar parejas más inmediatas que duraderas, cuyo resultado sigue siendo el mismo: el libre intercambio de orgasmos. De este modo, no subvierten las expectativas sociales sobre la exclusividad sexual monogámica, sino que, por el contrario, se evidencia la promiscuidad $y$ morbosidad como elementos apreciados y constitutivos de su vivenciar.
Con respecto a la socialización de la masculinidad oso en relación con otras masculinidades homosexuales, se presupone una situación de espejo consistente en que los estigmatizados son tan excluyentes como los que estigmatizan. Ello con afán de mostrar lo que deben soportar, pero también a modo de reflejo de la opresión que experimentan, buscan culpables y se cobran en cada oportunidad (Noh Poot, 2010).

Estos antecedentes abren una discusión sobre la necesidad de modificar estereotipos sociales que contribuyen a mantener relaciones que desfavorecen a un grupo social y que permanecen debido a la falta de reflexión. Este patrón automático es el que permite un enraizamiento profundo de las prácticas discriminatorias, pues se ampara en un discurso explícito que aboga por la igualdad de género y la no discriminación por la orientación sexual, lo que permite mantener una autoimagen positiva, pero que encubre el prejuicio (Cárdenas \& Barrientos, 2008).

Por otro lado, emergen percepciones polarizadas sobre la existencia o no de una subcultura oso en Santiago de Chile, pues, aunque se reconoce una masificación de la propuesta ursina y un cúmulo de sujetos que se reconocen a sí mismos como pertenecientes a ella, la segregación, autodiscriminación y el hacer de esta masculinidad homosexual un estilo de vida periférico limitan su incipiente consolidación. En consonancia con ello, resulta interesante que sus mismos adscritos definan las relaciones extragrupales como más sinceras y honestas, instancias donde su performance masculina se flexibiliza voluntaria y casi insoslayablemente, pese a que prefieran vínculos amistosos con sus símiles. 
Finalmente, el presente estudio cuestiona confluencias en los resultados dignas de atender, pero que escapan al objetivo del mismo y que pueden servir como interrogantes para futuras investigaciones, a saber: ¿qué motiva a los osos a reunirse si se perciben a sí mismos como sujetos altamente discriminadores, poco tolerantes y segmentarios?; la heteroidentificación de oso ¿propicia una identidad o una condición?; ¿reconocen la necesidad de una presencia política que los ubique en la palestra social para propiciar su consolidación como subcultura?

\section{Referencias}

Araya Andrade, K. \& Echeverría Chavarría, M. (1998). Los problemas sociales asociados a la homosexualidad masculina y las respuestas que se han generado en torno a las necesidades de esta población. (Tesis de Licenciatura, Universidad de Costa Rica, Ciudad Universitaria Rodrigo Facio, Costa Rica). Recuperado de http://www.ts.ucr.ac.cr/binarios/tfglic/tfg-1-1998-08.pdf

Barrientos, J. \& Cárdenas, M. (2013). Homofobia y calidad de vida de gay y lesbianas: una mirada psicosocial. Psykhe, 22(1), 3-14. http://dx.doi.org/10.7764/psykhe.22.1.553

Bermúdez, M. M. (2013). Connel y el concepto de masculinidades hegemónicas: notas críticas desde la obra de Pierre Bourdieu. Revista Estudios Feministas, 21(1), 283-300. http://dx.doi.org/10.1590/S0104-026X2013000100015

Blázquez, G. \& Liarte, A. (2013). Osos, locas y chongos. Masculinidades homosexuales en Córdoba. Trabajo presentado en el XXIX Congreso de la Asociación Latinoamericana de Sociología. Santiago, Chile. Recuperado de http://actacientifica.servicioit.cl/biblioteca/gt/GT11/GT11_BlazquezGLiarteA.pdf

Cárdenas, M. \& Barrientos, J. (2008). Actitudes explícitas e implícitas hacia los hombres homosexuales en una muestra de estudiantes universitarios en Chile. Psykhe, 17(2), 17-25. http://dx.doi.org/10.4067/S0718-22282008000200002

Carrigan, T., Connell, B., \& Lee, J. (1985). Toward a New Sociology of Masculinity. Theory and Society, 14(5), 551-604.

Clatterbaugh, K. (1997). Contemporary perspectives on masculinity: Men, women and politics in modern society. Boulder, Colorado: Westview Press.

Connell, R. W. (1987). Gender and power: Society, the person and sexual politics. Palo Alto, California: Standford University Press.

Dankhe, G. L. (1989). Investigación y comunicación. En C. Fernández-Collado \& G. L. Dankhe (Comps.), La comunicación humana: ciencia social (pp. 385-454). México, Distrito Federal, México: Ed. McGraw-Hill.

Delgado, J., Barrientos, E., Vega, A., Gutiérrez, K., Zaffirri, I., \& Ramírez, P. (2016). Identidad sexual en jóvenes gay del norte de Chile. Sexualidad, Salud y Sociedad, 23, 118-139.

https://dx.doi.org/10.1590/1984-6487.sess.2016.23.05.a 
Diez, C. (2003). Deporte, socialización y género. En X. Medina \& R. Sánchez (Ed.), Culturas en juego (pp. 297-324). Barcelona, España: Icaria.

Donahue, B. \& Stoner, J (22, diciembre, 2001). Recursos para osos. Los códigos de los osos [Website]. Recuperado de http://resourcesforbears.com/nbcs/es_index.html

Faur, E. (2004). Masculinidades y desarrollo social. Las relaciones de género desde una perspectiva de los hombres. Bogotá, Colombia: Arango Editores.

Flick, U. (2004). Introducción a la investigación cualitativa. Madrid, España: Morata.

Giddens, A. (1991). Sociología. Madrid, España: Editorial Alianza.

Gilligan, C. (1982). In a different voice. Cambridge, Massachusetts: Harvard University Press.

Gilmore, D. D. (1994). Hacerse hombre. Concepciones culturales de la masculinidad. Barcelona, España: Paidós.

Giménez, G. (1996). La identidad social o el retorno del sujeto en sociología. En L. Méndez y Mercado (Ed.), Identidad: análisis y teoría, simbolismo, sociedades complejas, nacionalismo y etnicidad. Trabajo presentado en III Coloquio Paul Kirchhoff. México, Distrito Federal, México.

Green, J. N. (1999). Além do carnaval: A homossexualidade masculine no Brasil de século $X X$. Sao Paulo, Brasil: UNESP.

Guasch, O. (2000). La crisis de la heterosexualidad. Barcelona, España: Laertes.

Gutiérrez, J. (2004). De osos, cachorros, daddies, chubbies, nutrias, lobos y chasers. Masculinidad, cuerpo e identidad entre varones gay del club de osos mexicanos. (Tesis de Licenciatura inédita). Escuela Nacional de Antropología e Historia, México.

Hardman, P. (1993). Homoaffectionalism: Male bonding from Gilgamesh to the present. San Francisco, California: GLB Publishers.

HAZLO.mx (30, junio, 2014). Masculinidad, piel y rudeza: subcultura leather [Blog]. Recuperado de

http://www.hazlo.mx/masculinidad-piel-y-rudeza-subcultura-leather

Hennen, P. (2005). Bear bodies, bear masculinity: Recuperation, resistance, or retreat? Gender and Society, 19(1), 25-43.

https://doi.org/10.1177/0891243204269408

Hernández, R., Fernández C., \& Baptista P. (1998). Metodología de la investigación. México, Distrito Federal, México: McGraw-Hill Interamericana Editores.

Iñiguez, L. \& Antaki, Ch. (1998). Análisis del discurso. Barcelona, España: Anthropos.

Kates, S. M. (1998). Twenty million new customers! Understanding gay men's consumer behavior. New York, New York: Harrington Park Press.

Kimmel, M. (1992). La producción teórica sobre la masculinidad: nuevos aportes. Ediciones de las Mujeres, 17, 129-138. Recuperado de https://is.gd/x7BMpc 
Kimmel, M. (1997). Homofobia, temor, vergüenza y silencio en la identidad masculina. Ediciones de las Mujeres, 24, 49-62. Recuperado de https://is.gd/Aq1Jwo

Kulka, S. B. (2000). Pragmática del discurso. En T. A. van Dijk (Ed.), El discurso como interacción social: estudios del discurso, introducción multidisciplinaria (pp. 67-100). Barcelona, España: Gedisa.

Lamas, M. (1996). El género: la construcción social de la diferencia sexual. México, Distrito Federal, México: PUEG-UNAM y Grupo Editorial Migue Ángel Porrúa.

Le Breton, D. (1995). Antropología del cuerpo y modernidad. Buenos Aires, Argentina: Nueva Visión.

Ley sobre protección de la vida privada, 19.628. (2012). Recuperado de http://bcn.cl/1uv2v

Ley sobre la investigación científica en el ser humano, su genoma y prohíbe la clonación humana, 20.120. (2013). Recuperado de http://bcn.cl/1uxxf

Marmor, J. (1980). Overview: The multiple roots of homosexual behavior. En J. Marmor (Ed.), Homosexual behavior: A modern reappraisal (3-22). New York, New York: Basic books.

Martín-Crespo Blanco, M. C. \& Salamanca Castro, A. B. (2007). El muestreo en la investigación cualitativa. Nure Investigación, 27, 1-4.

Mazzei, G. (1979). "Who's who in the zoo? A glossary of gay animals". The Advocate the National Gay \& Lesbian News Magazine, 42. Recuperado de http://www.advocate.com/comedy/2014/04/17/tbt-when-advocate-invented-bears

Movimiento Unificado de Minorías Sexuales. (2006). Informe de derechos humanos y discriminación. Santiago, Chile: MUMS.

Noh Poot, C. T. (2010). Masculinidades diversas: prácticas sexuales en jóvenes de Mérida, Yucatán. En G. Villagómez Valdés, E. M. Escoffié Aguilar, \& L. Vera Gamboa (Eds.), Varones y masculinidades en transformación (pp. 271-298). Recuperado de http://www.kookay.org/Masculinidades_Villag\%C3\%B3mez\%20Et\%20al_2010.pdf

Nunan, A. \& Jablonski, B. (2002). Homossexualidade e preconceito: Aspectos da subcultura homossexual no Rio de Janeiro. Arquivos Brasileiros de Psicología, 54(1), 21-32. Recuperado de http://aequipedoph.ufpa.br/arquivos/subculturahomossexual.pdf

Parker, L. (1998). Hacia una economía política del cuerpo: construcción de la masculinidad y de la homosexualidad masculina en Brasil. En T. Valdés \& J. Olavarría (Eds.), Masculinidades y equidad de género en América Latina (pp. 106-130). Santiago, Chile: Flacso/ NFPA.

Plummer, K. (1992). Modern homosexualities. London, United Kingdom: Routledge.

Sáez, J. (2005). Excesos de la masculinidad: la cultura leather y la cultura de los osos. En C. Romero Bachiller, S. García Dauder, \& C. Bargueiras Martínez (Eds.), El eje del mal es heterosexual. Figuraciones, movimientos y prácticas feministas queer (pp. 137-149). Madrid, España: Traficantes de Sueños. 
Sullivan, A. (1996). Praticamente normal (umadiscussão sobre homossexualidade). Sao Paulo, Brasil: Companhia das Letras.

Taylor, S. J. \& Bogdan R. (1986). Introducción a los métodos cualitativos de investigación. Buenos Aires, Argentina: Editorial Paidós.

Trevisan, J. S. (2000). Devassos no Paraíso: A homossexualidade no Brasil, da colônia à atualidade. Rio de Janeiro, Brasil: Record.

Viveros Vigoya, M. (2002). De quebradores y cumplidores: sobre hombres, masculinidades y relaciones de género en Colombia. Bogotá, Colombia: Universidad Nacional de Colombia.

Viveros Vigoya, M. \& Garay Ariza, G. (1998). Cuerpo, diferencias y desigualdades., M. \& Garay, G. (1998). Cuerpo, diferencias y desigualdades. Bogotá, Colombia: Universidad Nacional de Colombia.

Weeks, J. (1998). Sexualidad. México, Distrito Federal, México: Paidós.

Wright, L. (1997). Introduction: Theoretical bears. En J. Dececco \& L. Wright (Ed.), The bear book. Readings in the history and evolution of a gay male subculture (pp. 6-86). New York, New York: Harrington Park Press. 